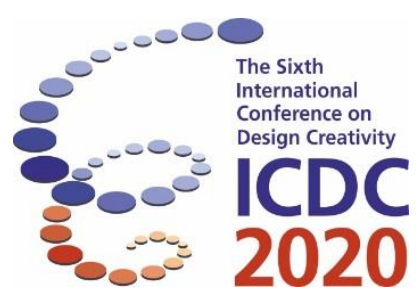

\title{
Creativity Assessment via Novelty and Usefulness (CANU) - Approach to an Easy to Use Objective Test Tool
}

\author{
Lorenz Prasch, Philipp Maruhn, Marcel Brünn and Klaus Bengler \\ Chair of Ergonomics, Technical University of Munich, Germany
}

\begin{abstract}
Creativity, or the ability to generate new and useful ideas or products, is considered a key factor in modern society. The role it plays in the workplace and day-to-day activities is on the rise and - in the light of recent advances in artificial intelligence - will continue to increase. Considering the importance of the subject at hand, the fact that there is no standardized, reliable and scalable measurement tool is astonishing. In this paper, we introduce the creativity assessment via novelty and usefulness (CANU), an approach to an easy-to-use online measurement tool for creative performance. Using specifically tailored tasks, we aim to assess participants' performance in regard to the novelty and usefulness of solutions they produce.
\end{abstract}

Keywords: creativity, measurement, user-centered design, tool

\section{Introduction}

Research on creativity, "humankind's ultimate resource" (Toynbee, 1964) or the ability that allowed our species to evolve from cave dwellers existing in hunter-gatherer societies to astronauts exploring space, living in an interconnected information society (Eagleman \& Brandt, 2017) is underrepresented (especially when compared to similar constructs like intelligence or personality) to this day (Batey, 2012). During the last century, the human ability to solve non-routine problems that require non-linear and creative thinking (Drucker, 1959; Reinhardt et al., 2011) has gained importance especially in the workplace (Ramírez \& Nembhard, 2004) and is projected to continue to do so (Bubb, 2006), especially in the context of past and recent advances in automation that can gradually support or substitute the human worker in a wider variety of cognitive tasks.

Creativity saw a rise in research interest following the address to the American Association of Psychologists by their then president, J.P. Guilford in 1950 and has since been studied by various disciplines (Batey, 2012). However, a lack of interdisciplinary research has led to a fragmented field (Hennessey \& Amabile, 2010). Despite several attempts to unify the construct, a clear definition of "what creativity is, and what it is not, hangs as the mythical albatross around the neck of scientific research on creativity" (Prentky, 2001). In the last decades there were many promising attempts to define creativity, however there is still no consensus reached (Plucker et al., 2004; Sarkar \& Chakrabarti, 2011).

\subsection{Human Creativity at Work will not be replaced by AI}

Especially since the first industrial revolution where fossil fuels were systematically used to harness energy on demand for the first time, work - and the society as a whole - has changed substantially (Bubb, 2006). This entailed employing Frederick Winslow Taylor's (1914) principles of scientific 
management, to reduce manual labor into small entities. These were analyzed, optimized and could eventually be automated, so humans could focus on more complex tasks. This resulted in a fifty-fold increase of productivity and has subsequently been suggested to be responsible for all economic and social gains that were achieved in the $20^{\text {th }}$ century (Drucker, 1999).

Considering the capability to increasingly substitute cognitive work using advanced computational techniques and artificial intelligence (AI), approximately $47 \%$ of employment in the entire United States is at risk for automation by means of computer-controlled equipment (Frey \& Osborne, 2017). This leads to the notion that jobs in the future will change massively towards the aspect of creative knowledge generation, since it still poses a major challenge for AI (Colton \& Wiggins, 2012). A primary goal of ergonomics is to support the population in diverse working contexts, aiming to achieve an optimal synthesis of human well-being and system-performance (Dul et al., 2012). This challenge has already been met fairly successful in regard to manual labor; the biggest challenge for the discipline in the 21st century is to achieve similar results for knowledge workers (Drucker, 1999). The main assets of knowledge workers are the acquisition, procession, generation and communication of knowledge (Drucker, 1959). Especially the generation of new knowledge requires creative thinking; thus, understanding and fostering creativity in diverse working conditions will be key for the successful application of ergonomics in knowledge workers.

\subsubsection{The Problem with Creativity}

Even after years of research there is no commonly agreed upon definition of creativity (Plucker et al., 2004). There are many different definitions in various domains that focus on a diverse range of aspects of creativity (Batey, 2012). For example, etymologically speaking "creativity is a noun naming the phenomenon in which a person communicates a new concept" (Rhodes, 1961). This implies that the result of creative activity is a perceivable entity (an idea or product). This approach can also be found in the definition synthesized by Plucker and colleagues (2004). The authors synthesized from 90 articles on creativity and narrowed down to the following definition:

"Creativity is the interaction among aptitude, process, and environment by which an individual or group produces a perceptible product that is both novel and useful as defined within a social context."

(Plucker et al., 2004)

This definition focuses on three components influencing the creation of a new and appropriate (Zeng et al., 2011) outcome, namely the prerequisites of the person that creates (aptitude), the way they get there (process), and the surroundings they are in (environment). This is consistent with the $4 \mathrm{P}^{\prime}$ s model proposed by Mel Rhodes (1961) and later formalized by Mark Batey (2012) in the equation:

$$
\text { person } \times \text { process } \times \text { press }^{1}=\text { product } \quad{ }^{1} \text { this refers to the environmental press }
$$

Moving forward from this, it seems reasonable to assess creativity from the point of the result or product of the creative endeavour. This is also in agreement with researchers from the design domain (Sarkar \& Chakrabarti, 2011). This product should be both novel as well as appropriate (Runco \& Jaeger, 2012) on a continuous scale (Piffer, 2012). Usefulness in this case seems to be an appropriate term for an entire cluster of characteristics found in many definitions (Sarkar \& Chakrabarti, 2008).Measuring usefulness or appropriateness, seems fairly straightforward (as a solution to a given problem can solve said problem to a certain, differentiable degree). However, when assessing novelty, researchers distinguish between creativity that is novel only in the mind of the person creating versus creativity that is novel to humanity in its entirety. Although the wording differs across different authors, the distinction remains consistent (Prasch \& Bengler, 2019). For example, Czikszentmihalyi (2014) speaks of personally and unqualifiedly creative individuals, Boden $(1998)$ refers to psychological $(P)$ and historical $(P)$ creativity and Gardner (1993) separates little-C and Big-C creativity. 


\subsection{Assessment Today lacks Scalability}

In the literature, there are different assessment methods varying in their approach (assessment of the person, the process, the press or the product (Amabile et al., 1996; Eysenck, 1993; Finke et al., 1992; Kaufman et al., 2008). However, they all have some sort of drawback, especially and most critically the fact that hardly any of them present practical and reliable scaling (Prasch \& Bengler, 2019).The often subjective ratings make current measurement tools resource-heavy and limit the comparability of results across different studies. This leads to a rather small amount of results in total which is far from ideal for fundamental research on a construct as complex as creativity. An approach that enables researchers to properly share and compare results, ideally one single database that encompasses all studies in a meaningful way, might be a proper basis to foster research on the topic.

Summarizing, there is a consensus that creativity involves the generation of novel and useful ideas and products (Amabile, 1983; Mumford, 2003). Moreover domain-specific (Sternberg et al., 2005) results of the creative endeavour are the most promising object to measure. On that theoretical basis, we propose a series of specifically tailored tasks that enable creative solutions and are comparable on a continuous scale in the two dimensions novelty and usefulness. This aims to enable a thorough investigation by participant studies to clarify which means influence creativity and how it can be facilitated at work.

\section{CANU-Test}

The test for creativity assessment via novelty and usefulness (CANU) was developed using a usercentered design method following the ISO 9241-210 (ISO, 2019) and is tailored to two types of users: Scientists and participants in scientific studies. The tool incorporates an in-depth interface for researchers enabling them to create, manage, and analyze studies as well as an interface for participants presenting solely the tasks to be accomplished.

\subsection{Task Design}

The tasks implemented in this measuring instrument have been designed to best meet the diverse requirements of both the human and computer system for such an application. Taking into account the division of the evaluation into novelty and usefulness, the tasks have to fulfil certain criteria. To assess novelty, any given solution can be related back to all solutions collected. The given solution is compared with the set of already collected solutions and if there is a match, a novelty value for the current solution can be assigned depending on the number of identical solutions in the set: The more often a certain solution is found for a task, the less novel it is and vice versa. The main challenge in developing creativity tasks is to set them in such a way that they can be evaluated by a machine in terms of usefulness. In contrast to human evaluators, a computer needs certain conditions, on the basis of which it can assign a usefulness value to a solution. In order to classify the solutions, a maximum and a minimum useful solution must be defined for each task. Usefulness is regarded as the degree to which the task has been fulfilled. It is therefore necessary to create evaluation criteria by means of which a computer can determine whether one solution is more useful than another (and by how much). Within these evaluation criteria lies the difficulty of the development of automatically evaluable tasks. After all, creativity tasks should have as open a solution space as possible and not be restricted by conditions to the solution, since an open solution space is mandatory in order to explore and generate solutions beyond the obvious. Therefore, an attempt was made to connect the human requirement for a sufficiently large solution space with the computational requirements for a limited solution space with discrete values. Discrete solution spaces ensure machine-readability and the efficient calculation of creativity metrics. In principle, the usefulness value for all task types is formed by a relation between an achieved number of criteria and a maximum possible number of attainable criteria. In the context of this instrument, two distinct tasks have been developed: Blocks and New Words. When considering the tasks, it was important to identify the essential patterns of thought that should lead to a creative solution, such as a change of perspective, leaving mental boundaries or association. Blocks is a visually constructive task that instructs participants to fill out a given shape with different tetrominoes (cf. Figure 1 left). The shape has to be filled in different ways and in the best possible way, i.e. covering all darkened squares. Each element type can be used as often as desired to fill the shape. A solution of this task is represented by a matrix whose entries encode the position of the elements on the field. The most useful solution 
would be to completely fill the form within the form boundary. In this case, no square of a tetromino would be outside the shape. Before processing, the field matrix has the numeric values of 0 outside the shape boundary and 1 within the boundary. By adding the tetrominoes, whose squares are assigned a letter to identify the shape used, the value of a square of the playing field is increased altered. This way, the resulting graphic can be made readable for the computer. New Words (cf. Figure 1 right) is a verbal constructive task that involves forming new words from a given set of letters. The given letters can, but do not have to, form meaningful words. Each instance of an occurring letter may only be used once in the newly formed words. The specification of several letter or word sets ensures a sufficiently large solution space. In this task, the usefulness dimension is determined by comparing the solution words to a list of valid words (see section usefulness). If a given solution word is found in this list, it is a valid solution. Words that are not in the list will be considered incorrect. The number of letters used in the correct words is then evaluated and related to the number of the given letters. As a further feature, the tasks should be easily configurable by varying the exact execution. This means that several task variants should be able to emerge from one task type. Therefore, the shapes in Blocks and the sets of letters in New Words change with each completed subtask on the basis of ready-made configurations. Each subtask is to be completed in 180 seconds before the application forwards the participant to the next task. The time limitation ensures a reasonable processing time as well as comparable results through a standardized study execution.

In summary, the tasks have been designed to provide sufficient opportunities to be creative, while at the same time ensuring automatic evaluation by a computer.

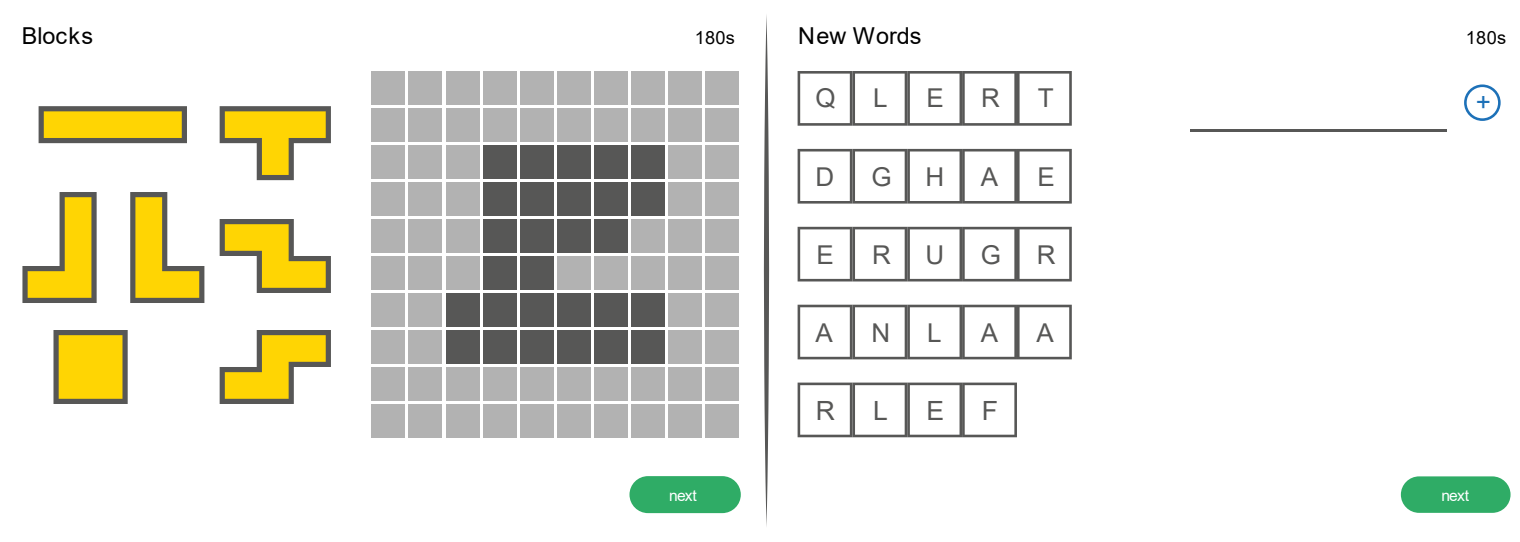

Figure 1. Layout of the visual constructive task (left). Participants need to fill the darker squares on the right with the building blocks on the left as best as possible. Building blocks can be rotated by right-clicking them. Layout of the verbal constructive task (right). Participants need to recombine the letters to the left into new words. The system automatically detects if a certain letter has already been used, reduces its opacity and prevents the user from filling it into the form on the right.

\subsection{Front-End}

\subsubsection{Researchers}

The typical workflow for study generation is held as simple as possible. After setting up an account on the server, researchers can easily add studies via a button click. The study needs a name for identification, the necessary number of individual tasks (Blocks and New Words) as well as the number of trial groups (e.g. baseline, condition 1 and condition 2). A number of unique links equal to the number of groups will be generated and can be distributed freely. When conducting a study, the links for specific study groups can either be distributed via the internet or be presented in a controlled setting on a PC.

On a researcher dashboard a quick overview of currently open as well as already closed studies is displayed with a quick info on the number of completed questionnaires. Selecting a study will lead to its dashboard view, where a short summary of the study itself, a descriptive overview of collected data as well as a data download can be found. 


\subsubsection{Participants}

Participants see a simplistic interface and will get a short introduction to the task, including a video showcasing possible interactions. Following this, the tasks selected by the researcher (cf. Figure 1) will be displayed in random order. After finishing the study, participants are forwarded to a thank you page.

\subsection{Back-End}

The back-end is implemented using a MongoDB database and has a structure that builds mainly upon the two central collections solutions and unique solutions. Every solution that is submitted triggers an entry into the solution database. This entry includes a machine-readable representation of the solution itself, the participant id, study and group it belongs to, the task that was fulfilled and a time stamp. Additionally, every solution is represented by an entry in the unique solutions database. This database only contains one entry for every solution instance plus a counter showing how often this specific solution has already been submitted. If no such entry exists, the given solution is truly novel for the database and a new entry is generated containing a machine-readable representation of the solution, the task it belongs to, the counter of identical solutions (equals one in case of the initial entry) as well as its novelty and usefulness values. The calculation of these two indicators is described in further detail in section 2.4.

This means, if an identical solution has already been submitted (by any participant of any study conducted), there is already an existing entry. In this case, the counter for this specific solution is incremented by one and the novelty value is re-evaluated using the updated numbers.

\subsection{Score Calculation}

Solutions are scored in two separate dimensions, novelty and usefulness, each ranging between 0 (not at all novel/useful) and 1 (perfect).

\subsubsection{Usefulness}

Usefulness is determined by how successful participants completed the given task. The numeric values between 0 (not successful at all) and 1 (perfect solution) are calculated using the quotient between accurately used building blocks/letters and maximum building blocks/letters in a perfect solution. For the two tasks implemented, this can be expressed in an equation respectively.

Blocks. A solution to the blocks task is the more useful, the more of the dark squares (cf. Figure 1) are properly filled with tetrominoes. As every solution has an inherent maximum, the quotient can be calculated as follows:

$$
\text { Usefulness }=\frac{N_{\text {covered }}}{N_{\max }}
$$

where $N_{\text {covered }}$ is the number of squares in the form that are covered by a tetromino and $N_{\max }$ is the number of squares within the form.

New Words. A solution to the new words task is the more useful, the more of the letters provided (cf. Figure 1) have been constructed into new, valid words. The validity of a certain word is checked by comparing it to an array of approx. 275,000 English words (available via npm or GitHub, https://github.com/words/an-array-of-english-words). If the word in the solution can be found within the array, all letters within said word are counted as valid. Because only a certain number of letters is provided, the maximum value is fixed. This results in the following equation:

$$
\text { Usefulness }=\frac{N_{\text {valid }}}{N_{\max }}
$$

where $N_{\text {valid }}$ is the number of letters used in valid English words and $N_{\max }$ is the total number of available letters. 


\subsubsection{Novelty}

Novelty is task independent. How novel a solution is, depends on how original it is. This means, the more participants already came up with the same solution, the less novel a specific solution is considered. The numeric values between 0 (all participants have the identical solution) and 1 (a unique solution) are calculated using the quotient between the number of identical solutions and total number of solutions to a given task. In mathematical terms this can be expressed as follows:

$$
f(n, N)=\left\{\begin{array}{l}
1, \text { if } n=1 \\
\frac{N-n}{N}, \text { else }
\end{array}\right.
$$

Where $N$ is the total number of solutions submitted for the selected task, and $n$ is the number of solutions identical to the one provided.

To illustrate the scoring method, figure 2 shows an evaluation for an exemplary Blocks task.

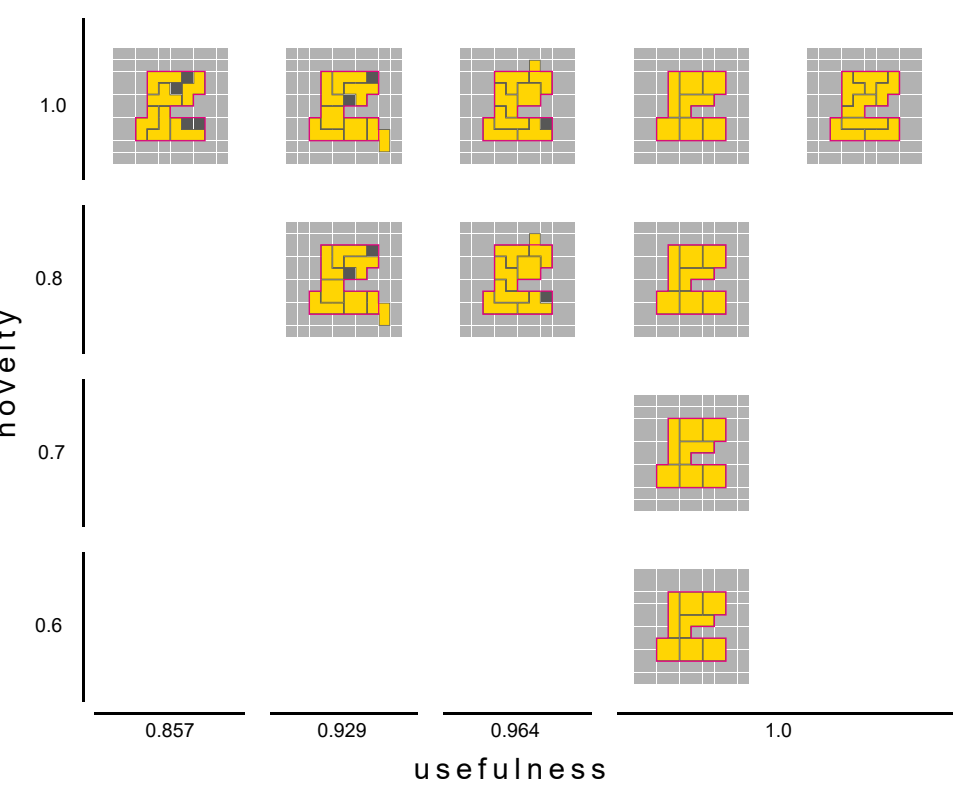

Figure 2. Example of novelty and usefulness assessment. The total often provided solutions can be judged with four novelty values depending on their number of occurrence and four usefulness values, depending on how many of the darkened squares are covered by a tetrominoe.

\subsection{Modular Structure}

In order to make the tool as expandable as possible and ensure the ability to cope with domain specific tasks (Mumford, 2003), a simple interface was implemented. This is to ensure that additional creativity tasks can be easily integrated into the existing application. For this, tasks must meet a number of requirements. First and foremost, the task must be oriented towards machine readability. Both machinereadable input mechanisms as well as fixed evaluation criteria, by means of which the degree of fulfilment of the task can be determined, must be given in order to obtain meaningful results. This includes the presence of a perfect and a worst solution. In addition, it should be possible to create multiple configurations, or start conditions encoded in an array from the same task type.

\section{Summary and Limitations}

The measurement tool introduced in this paper aims to overcome the shortcomings of existing tests for creativity by making its evaluation entirely dependent on the result a participant produces and automatically scoring it in mathematical terms. In order to meet these requirements, two tasks, Blocks and New Words, were implemented. Both tasks leave sufficient room for creative problem-solving while 
being machine-readable to ensure automatic rating capability. However, creating and automatically analysing unexpected results is not possible in the current implementation. Future iterations could introduce more freedom of use. Even though some researchers believe that creativity can never be measured by objective analysis alone, since the perception of creativity always seems to depend on the evaluator's accumulated knowledge and sociocultural context (Amabile, 2018; Baer, 2016), we believe the objective rating of creativity to be one of the major advantages of the CANU-test.

The test relies heavily on a multitude of solutions in order to be able to sufficiently judge a specific solution's novelty or originality. This has the disadvantage that initial results may not represent a proper measurement; however, ensuring that results will improve over time. Furthermore, we can not be certain that novelty and usefulness are indeed the only two dimensions comprising a creative solution. Some authors argue that impact is relevant as well when it comes to creative output in the real world (Piffer, 2012). However, we adapt the view that impact differentiates between the constructs creativity and innovation and hence disregarded this dimension (Dul \& Ceylan, 2014). Additionally, it is reasonable to assume impact as a creativity assessment in itself (if the notion that more creative solutions tend to have a higher impact than non creative ones is true) (Prasch \& Bengler, 2019).

As of now, the CANU-test only includes two tasks. This poses the potential danger that only very specific types of creativity can be assessed. Due to the modular structure of the test however, additional task-types are possible in the future. For instance, machine learning could be used to understand and cluster solutions that are generated from more complex problems with higher similarity to the real world. An additional drawback is that only a type of task to which a perfect solution in terms of usefulness exists can be applied in this setting. These types of tasks can be classified between puzzle tasks on the one hand and completely open ideation tasks (e.g. novel uses of a paper clip) on the other. Thus, they solve the measurement problem of the latter while keeping their potential for creative problem solving. However, empirical evidence of the method's validity still needs to be tested.

\section{Conclusion}

The CANU can provide a basis for a database of objective and comparable solutions to creative problem solving. It would enable researchers to conduct studies and meta-analyses in order to better understand the complex construct and central human capability that is creativity. Moreover, it provides an opportunity for research on how automated systems (e.g. some sort of solution helper) should interact with humans in order to enable them to produce more novel and/or useful solutions. These insights can offer a steppingstone towards meaningful automation that does not rob humanity of its capabilities but rather assists in freeing cognitive capacities for truly creative tasks.

The CANU is publicly accessible via https://creativity.lfe.mw.tum.de/

\section{References}

Amabile, T. M. (1983). The social psychology of creativity: A componential conceptualization. Journal of Personality and Social Psychology, 45(2), 357.

Amabile, T. M. (2018). Creativity in context: Update to the social psychology of creativity. Routledge.

Amabile, T. M., Conti, R., Coon, H., Lazenby, J., \& Herron, M. (1996). Assesing the Work Environment for Creativity. In Academy of Management Journal (Vol. 39, Issue 5, pp. 1154-1184). https://doi.org/10.2307/256995 Baer, J. (2016). Creativity Doesn't Develop in a Vacuum. New Directions for Child and Adolescent Development, 2016(151), 9-20. https://doi.org/10.1002/cad.20151

Batey, M. (2012). The Measurement of Creativity: From Definitional Consensus to the Introduction of a New Heuristic Framework. Creativity Research Journal, 24(1), 95-104. https://doi.org/10.1080/10400419.2012.649181

Boden, M. A. (1998). Creativity and artificial intelligence. Artificial Intelligence, 103(1-2), 347-356. https://doi.org/10.1016/S0004-3702(98)00055-1

Bubb, H. (2006). A consideration of the nature of work and the consequences for the human-oriented design of production and products. Applied Ergonomics, 37(4 SPEC. ISS.), 401-407. https://doi.org/10.1016/j.apergo.2006.04.004

Colton, S., \& Wiggins, G. A. (2012). Computational creativity: The final frontier? Frontiers in Artificial Intelligence and Applications, 242, 21-26. https://doi.org/10.3233/978-1-61499-098-7-21 
Csikszentmihalyi, M. (2014). Society, Culture, and Person: A Systems View of Creativity. In The Systems Model of Creativity: The Collected Works of Mihaly Csikszentmihalyi (pp. 47-61). Springer Netherlands. https://doi.org/10.1007/978-94-017-9085-7_4

Drucker, P. F. (1959). Landmarks of Tomorrow: A Report on the New “Post-Modern” World. Harper \& Row. Drucker, P. F. (1999). Knowledge Worker Productivity: The Biggest Challenge. California Management Review, 41(2), 79-85.

Dul, J., Bruder, R., Buckle, P., Carayon, P., Falzon, P., Marras, W. S., Wilson, J. R., \& van der Doelen, B. (2012). A strategy for human factors/ergonomics: developing the discipline and profession. Ergonomics, 55(4), 377-395. https://doi.org/10.1080/00140139.2012.661087

Dul, J., \& Ceylan, C. (2014). The impact of a creativity-supporting work environment on a firm's product innovation performance. Journal of Product Innovation Management, 31(6), 1254-1267. https://doi.org/10.1111/jpim.12149

Eagleman, D., \& Brandt, A. (2017). The runaway species: How human creativity remakes the world. Catapult. Eysenck, H. J. (1993). Creativity and personality: Suggestions for a theory. Psychological Inquiry, 4(3), $147-178$. Finke, R. A., Ward, T. B., \& Smith, S. M. (1992). Creative cognition: Theory, research, and applications.

Frey, C. B., \& Osborne, M. A. (2017). The future of employment: How susceptible are jobs to computerisation? Technological Forecasting and Social Change, 114, 254-280. https://doi.org/10.1016/j.techfore.2016.08.019

Gardner, H. (1993). Creating minds: an anatomy as seenthrough the lives of Freud, Einstein, Picasso, Stravinsky, Eliot, Graham and Gandhi. HarperCollinsPublishers.

Guilford, J. P. (1950). Creativity. The American Psychologist, 5(9), 444-454. https://doi.org/10.1037/h0063487 Hennessey, B. A., \& Amabile, T. M. (2010). Creativity (2010 Annual Review of Psychology). Annual Review of Psychology, 61, 569-598. https://doi.org/10.1146/annurev.psych.093008.100416

ISO. (2019). Ergonomics of Human-System Interaction -- Part 210: Human-Centered Design for Interactive Systems (Vol. 2019).

Kaufman, J. C., Plucker, J. A., \& Baer, J. (2008). Essentials of creativity assessment (Vol. 53). John Wiley \& Sons. Mumford, M. D. (2003). Where Have We Been, Where Are We Going? Taking Stock in Creativity Research. Creativity Research Journal, 15(2-3), 107-120. https://doi.org/10.1080/10400419.2003.9651403

Piffer, D. (2012). Can creativity be measured? An attempt to clarify the notion of creativity and general directions for future research. Thinking Skills and Creativity, 7(3), 258-264. https://doi.org/10.1016/j.tsc.2012.04.009

Plucker, J. A., Beghetto, R. A., \& Dow, G. T. (2004). Why Isn't Creativity More Important to Educational Psychologists? Potentials, Pitfalls, and Future Directions in Creativity Research. Educational Psychologist, 39(2), 83-96. https://doi.org/10.1207/s15326985ep3902_1

Prasch, L., \& Bengler, K. (2019). Ergonomics in the Age of Creative Knowledge Workers -- Define, Assess, Optimize. In S. Bagnara, R. Tartaglia, S. Albolino, T. Alexander, \& Y. Fujita (Eds.), Proceedings of the 20th Congress of the International Ergonomics Association (IEA 2018) (Vol. 821, pp. 349-357). Springer International Publishing. https://doi.org/10.1007/978-3-319-96080-7_41

Prentky, R. A. (2001). Mental Illness and Roots of Genius. Creativity Research Journal, 13(1), 95-104. https://doi.org/10.1207/S15326934CRJ1301_11

Ramírez, Y. W., \& Nembhard, D. A. (2004). Measuring knowledge worker productivity. Journal of Intellectual Capital, 5(4), 602-628. https://doi.org/10.1108/14691930410567040

Reinhardt, W., Schmidt, B., Sloep, P., \& Drachsler, H. (2011). Knowledge Worker Roles and Actions-Results of Two Empirical Studies. Knowledge and Process Management, 18(3), 150-174. https://doi.org/10.1002/kpm.378 Rhodes, M. (1961). An Analysis of Creativity. The Phi Delta Kappan, 42(7), 305-310. https://doi.org/10.2307/20342603

Runco, M. A., \& Jaeger, G. J. (2012). The Standard Definition of Creativity. Creativity Research Journal, 24(1), 92-96. https://doi.org/10.1080/10400419.2012.650092

Sarkar, P., \& Chakrabarti, A. (2008). Studying engineering design creativity. Workshop on Studying Design Creativity.

Sarkar, P., \& Chakrabarti, A. (2011). Assessing design creativity. Design Studies, 32(4), 348-383.

Sternberg, R. J., Todd, L. I., Kaufman, J. C., \& Pretz, J. E. (2005). Creativity. In K. J. Holyoak \& R. G. Morrison (Eds.), The Cambridge Handbook of Thinking and Reasoning (pp. 351--369). Cambridge University Press.

Taylor, F. W. (1914). The Principles of Scientific Management. Harper \& Brothers.

Toynbee, A. (1964). Is America neglecting her creative minority? Widening Horizons in Creativity, 3-9.

Zeng, L., Proctor, R. W., \& Salvendy, G. (2011). Can traditional divergent thinking tests be trusted in measuring and predicting real-world creativity? Creativity Research Journal, 23(1), 24-37. https://doi.org/10.1080/10400419.2011.545713 